\title{
Parenteral Sustained Release Lipid Phase-Transition System of Ziprasidone: Fabrication and Evaluation for Schizophrenia Therapy
}

This article was published in the following Dove Press journal:

Drug Design, Development and Therapy

\author{
Urooj A Khan' \\ Uzma Parveen ${ }^{2}$ \\ Nazeer Hasan' \\ Mohammad Zubair Ahmed' \\ Suma Saad' \\ Farhan J Ahmad' \\ Gaurav K Jain'
}

'Nanoformulation Research Laboratory, Department of Pharmaceutics, School of Pharmaceutical Education and Research, Jamia Hamdard, New Delhi I I0062, India; ${ }^{2}$ Department of Moalejat, School of Unani Medical Education and Research, Jamia Hamdard, New Delhi I 10062, India
Correspondence: Gaurav K Jain Nanoformulation Research Laboratory, Department of Pharmaceutics, School of Pharmaceutical Education and Research, Jamia Hamdard, New Delhi I I0062, India, $\mathrm{Tel}+919811127909$

Fax +9| II 26059663

Email drgkjain@gmail.com
Introduction: Ziprasidone (ZP) is a novel atypical antipsychotic agent effective in the treatment of positive and negative symptoms of schizophrenia with low chances for extrapyramidal side effects (EPs) and cognitive deficits. ZP possesses poor oral bioavailability $(\sim 50 \%)$, short biological half-life $(\sim 2.5 \mathrm{~h})$ and due to extensive first-pass metabolism, a repeated dose is administered which makes the therapy non-adherent, leading to patient non-compliance. Therefore, this is a first report of developing parenteral ZP loaded sustained release phospholipid based phase-transition system (ZP-LPS).

Methods: The ZP-LPS system was formulated by mixing of biocompatible materials including phospholipid E 80, medium chain triglyceride (MCT) and ethanol. Optimization was done by aqueous titration method using pseudo-ternary phase diagram and dynamic rheological measurements. In vivo depot formation was confirmed by gamma scintigraphy after subcutaneous injection. Biodegradation and biocompatibility studies were performed for its safety evaluation. Finally, the efficacy of the formulation was assessed by Morris water maze (MWM) test and dizocilpine (MK-801) was used to induce schizophrenia in Sprague-Dawley rats.

Results: Optimized ZP-LPS showed rapid gelation ( $2 \mathrm{~min}$ ), highest change in viscosity ( $\sim 8000 \mathrm{mPa} . \mathrm{s}$ ) and sustained release of ZP over a period of 1 month. Gamma scintigraphy depicted that the low-viscosity ZP-LPS system undergo rapid in situ gelation. Biodegradation and biocompatibility studies revealed gradual degradation in size of depot over a period of 28 days without any inflammation at the injection site. In MWM test, escape latency, time spent and total distance in target quadrant were significantly improved $(\mathrm{p}<0.001)$ in the ZP-LPS group in comparison to the MK-801 group when evaluated at day 0 , day 7 and day 28 . However, significant improvement $(\mathrm{p}<0.001)$ was observed only at day 0 in ZP suspension group.

Conclusion: The overall result indicates that the novel ZP-LPS system is safe, biodegradable, and effective for the management of schizophrenia.

Keywords: Gamma scintigraphy, lipid phase-transition, Morris Water Maze (MWM) test, phospholipid, schizophrenia, ziprasidone (ZP)

\section{Introduction}

Schizophrenia is a chronic and devastating psychotic disease, often coupled with cycles of remission and relapse. Characterized by hallucinations, delusions, and other cognitive difficulties, schizophrenia can often be a lifelong struggle. ${ }^{1}$ Atypical antipsychotics, also termed as second generation antipsychotics, introduced in the 1980s had led to substantial improvements in the treatment of schizophrenia. 
Atypical antipsychotics, effective for the positive symptoms of schizophrenia, demonstrated a lack of negative symptoms resulting in enhanced efficacy and reduced side effects. ${ }^{2}$

Ziprasidone (ZP) is a novel atypical antipsychotic agent effective in the treatment of positive, negative, and affective symptoms of schizophrenia with a low chances for EPs, weight gain, and cognitive deficits. ${ }^{3}$ It is a serotonin dopamine antagonist, which shows highly selective and potent antagonistic activities for $\mathrm{D}_{2}$ and $5-\mathrm{HT}_{2}$ receptors. ${ }^{4}$ Additionally, it has high affinity for the $5-\mathrm{HT}_{1 \alpha}, 5-\mathrm{HT}_{1 \mathrm{~d}}$ and $5 \mathrm{HT}_{2 \mathrm{c}}$ receptors subtypes that could contribute to composite therapeutic effect. ${ }^{4,5}$

Currently, ZP is commercially available as an oral tablet and intramuscular injection formulation. ${ }^{6} \mathrm{ZP}$ possesses poor oral bioavailability $(\sim 60 \%)$ and short biological half-life $(\sim 2.5 \mathrm{~h})$ owing to extensive first-pass metabolism. ${ }^{7}$ Though the oral route offers advantages in terms of non-invasive therapy and ease of administration, the injectable ZP formulation possesses a number of advantages, including avoidance of first-pass metabolism, higher bioavailability, food-effect and the certainty of delivery of the therapeutic agent. Nevertheless, both the oral and intramuscular injection formulation of ZP must be taken daily making the therapy less effective, primarily due to non-adherence to long treatment cycles. ${ }^{8,9}$ Infrequent intake of the medication is far more common than complete non-adherence to therapy, posing a significant challenge. Incidences of relapse in schizophrenic patients lead to a reversal of gains achieved during therapy, loss of confidence, and loss of function. ${ }^{10}$ Further, re-hospitalization of relapsed patients carries both a large economic and personal cost. ${ }^{11}$ The ideal ZP delivery system would avoid the disadvantages of the current oral formulation and maintain plasma concentrations of the drug for a long time after a single administration. Thus, continuous delivery of ZP through a sustained release injection is an effective way to ensure adherence to therapy. Such a release may become possible using an injectable lipid phase-transition system (LPS). After subcutaneous injection, when in contact with aqueous body fluids, LPS undergoes a phase transition, forming a depot at the injection site. LPS can encapsulate and sustain the release of drugs of varying size and physico-chemical characteristics, and the lipids in LPS are biodegradable. ${ }^{12,13}$ The purpose of the present study was to develop a ZP loaded LPS system (ZP-LPS) to provide sustained release over prolong period of time.
This study appears to be the first report of sustainedrelease ZP injection. The rheological behavior and in vitro release of ZP-LPS was evaluated. Subsequently, the biocompatibility, biodegradability, depot formation, pharmacokinetic and pharmacodynamic properties of ZPLPS formulation after subcutaneous injection was investigated in animals.

\section{Materials and Methods Materials}

ZP was obtained as a gift sample from Jubilant Generics Limited, India. Phospholipid E 80 (Lipoid E 80) was purchased from Lipoid Co. Ltd (Ludwigshafen, Germany). Medium chain triglyceride (MCT) was generously obtained as gift sample from Abitec Corporation, USA. MK-801 (Dizocilpine) was purchased from Sigma Aldrich, USA. HPLC grade acetonitrile and water were purchased from Merck and Loba (Chemie), India respectively. Animal studies were performed following the approval of the protocol from the Institutional Animal Ethics Committee (IAEC) of Jamia Hamdard, New Delhi, in consensus with the Committee for the Purpose of Control and Supervision of Experiments on Animals' (CPCSEA) guidelines.

\section{Development and Optimization of ZP-LPS}

The LPS were developed by modification of the method previously reported in the literature. ${ }^{14}$ Briefly, ZP, phospholipid E 80 (PE80) and MCT were added to $100 \mathrm{mg}$ of ethanol and stirred continuously for $30 \mathrm{~min}$. The ethanolic mixture obtained was optimized for concentration of PE80 (varied from $400 \mathrm{mg}$ to $700 \mathrm{mg}$ ) and MCT (varied from $150 \mathrm{mg}$ to $350 \mathrm{mg}$ ) by aqueous titration method. Briefly, the ethanolic mixture with varied concentrations of P E80 and MCT was mixed properly and titrated with double distilled water. The microtitration was visually assessed for clarity or semi solid gel. The change in viscosity is plotted as a pseudo-ternary phase diagram. The changes in viscosity from liquid to semisolid gel upon the addition of a minimum quantity of water and the amount of drug release at day one were considered as optimization parameters for the ZP-LPS formulation.

\section{Rheological Studies of ZP-LPS Viscosity Measurements}

Brookfield R/S plus rheometer (Brookfield Engineering Laboratories, Inc, Middleboro, USA) was used to measure the viscosity of ZP-LPS using a C50-1 spindle in triplicate 
mode. The rotating speed was set up before measurement, to make sure the torque value in the limit of $10-100 \%$. During all experiments $37^{\circ} \mathrm{C}$ temperature was maintained. ${ }^{13}$

\section{Dynamic Rheological Measurements}

Rheology was performed using Brookfield R/S plus rheometer (Brookfield Engineering Laboratories, Inc, Middleboro, USA). Ten milligrams of PBS ( $\mathrm{pH} 7.4$ ) was added to $100 \mathrm{mg}$ of formulation and viscosity of the sample was measured with respect to time at constant value of frequency of $0.1 \mathrm{~Hz}$ varying shear stress values from 0.1 to $200 \mathrm{~Pa}$. All the experiments were performed in triplicate. $^{12}$

\section{Solubility Study of ZP-LPS}

For determination of solubility, the excess amount of ZPLPS formulation was added to $1.0 \mathrm{~mL}$ of PBS ( $\mathrm{pH} 7.4$ ) and kept on shaker water bath at $37^{\circ} \mathrm{C}$ for $72 \mathrm{~h}$ for equilibration. The formulation was analyzed by the HPLC method (as described in drug release section) following filtration by 0.22 micronPES syringe filter (Sigma-Aldrich, USA). The comparison was made with pure ZP.

\section{Drug Release of ZP-LPS}

The drug release behavior of ZP-LPS was determined by a dialysis method using PBS ( $\mathrm{pH} 7.4$ ) containing $1 \%$ Tween 20 as dissolution medium. A comparison was done with ZP suspension. ZP-LPS (equivalent to $20 \mathrm{mg}$ ZP) or ZP (20 mg) was suspended in $2.0 \mathrm{~mL}$ of dissolution medium contained in a dialysis bag (MWCO: $12000 \mathrm{~g} / \mathrm{mol}$ ). The dialysis bag was then suspended in a $200 \mathrm{~mL}$ glass beaker containing $100 \mathrm{~mL}$ of dissolution medium under mild agitation in a water bath at $37^{\circ} \mathrm{C}$. At pre-designated time points for up to 30 days, the dissolution medium $(0.5 \mathrm{~mL})$ was withdrawn following the addition of the same volume of the fresh medium. The analyses of the samples were done using the previously published HPLC method following slight modification and re-validation. ${ }^{15}$ Analysis was done using 5 $\mu \mathrm{m}$ RP 18 (C18), Lichrospher $^{\circledR} 100,(250 \times 4.6 \mathrm{~mm})$ attached to an HPLC equipped with variable wavelength programmable UV/VIS detector, quaternary LC-10 AT VP pump, SCL 10A VP system controller (Shimadzu), SPD-10 AVP column oven (Shimadzu), and a Rheodyne injector with a $20 \mu \mathrm{L}$ loop. For the drug analysis class-VP 5.032 software was used. Process parameters used for separation were as follows: temperature, $25 \pm 2{ }^{\circ} \mathrm{C}$; mobile phase, phosphate buffer and acetonitrile at $65: 35(\mathrm{v} / \mathrm{v})$; flow rate, $1.0 \mathrm{~mL} / \mathrm{min} ; \mathrm{pH}, 3$; and detection wavelength of ZP, 318 nm.All experiments were performed in triplicate.

\section{In vivo Biodegradation and Biocompatibility of ZP-LPS}

To assess the in vivo biodegradation of ZP-LPS the animals were divided into two groups: (A) examination at Day 0 and (B) examination at Day 28. The rats were injected with $0.3 \mathrm{~mL}$ of ZP-LPS solution subcutaneously and following depot formation, Day 0 group animals were sacrificed, the skin was surgically removed and the dimensions of the depot were measured by vernier caliper. Similar studies were performed at Day 28 for group B animals. A comparison between the data was made to understand biodegradation and biocompatibility of ZP-LPS.

\section{In vivo Depot Formation of ZP-LPS by Gamma Scintigraphy}

Gamma scintigraphy was carried out to confirm the development of sustained release depot at the site of injection following the administration of liquid ZP-LPS formulation. Briefly, $\mathrm{ZP}$ was radiolabeled with ${ }^{99 \mathrm{~m}}$ Technetium-pertechnetate (Tc$99 \mathrm{M})$ by an in-house developed standard procedure using stannous chloride as reducing agent. Labeling efficiency and stability was confirmed by instant thin layer chromatography. ${ }^{16}$ ZP-LPS Formulation was then developed using Tc-99M labeled ZP. ZP-LPS formulation $(0.3 \mathrm{~mL})$ containing $60 \mu \mathrm{Ci}$ ofTc-99Mwas then injected subcutaneously at the back of the three Sprague-Dawley rats. At pre-designated time points, $15 \mathrm{~min}, 6 \mathrm{~h}$ and $12 \mathrm{~h}$, gamma images were captured by gamma camera (Siemens T2 SPECT-CT) to understand drug release and depot formation. ${ }^{17}$

\section{In vivo Pharmacokinetic Study of ZP-LPS}

Pharmacokinetic study (PK) was performed in 12 SpragueDawley male rats (weighing approx. $250 \mathrm{mg}$ ). The animals were divided into 2 groups each containing 6 rats. A dose of $0.3 \mathrm{~mL}$ of optimized ZP-LPS was injected subcutaneously into back of rats of group I $(n=6)$ whereas group II received $0.5 \mathrm{~mL} \mathrm{ZP}$ suspension (equivalent to $100 \mathrm{mg}$ of $\mathrm{ZP}$ ) subcutaneously into back $(n=6)$. After the anesthetization of the rats using diethyl ether, the blood samples were collected into sodium-heparin tubes from the retro-orbital plexus at predetermined time intervals, i.e., 0.01, 0.02, 0.03, 0.04, 0.06, $0.08,0.16,0.25,0.5,1,1.5,2,3,6,9,12,14,21$, and 28 days. Plasma was separated out using REMI centrifuge at $5000 \mathrm{rpm}$ for $10 \mathrm{~min}$ and stored at $-20^{\circ} \mathrm{C}$ till analysis. The 
concentration of ZP was analyzed by the developed UPLCMS/MS method. The C-18 column (Waters ACQUITY UPLCTM BEH) with particle size of $1.7 \mu \mathrm{m}$ and having dimension $2.1 \mathrm{~mm} \times 100 \mathrm{~mm}$ was used in chromatographic conditions. LC-MS grade Degassed acetonitrile and $5 \mathrm{mM}$ ammonium acetate $(80: 20 \mathrm{v} / \mathrm{v})$ was used as mobile phase with flow rate of $0.4 \mathrm{~mL} / \mathrm{min}$ (gradient flow) and injection volume of $5 \mu \mathrm{L} / \mathrm{min}$. The run time for each sample was kept at $4.0 \mathrm{~min}$. The quantization was done by using Waters XEVO-TQD (MA, USA) (triple quadrupole) mass spectrometer (Micromass MS Technologies, Manchester, UK, and QCA896) under an electrospray ionization (ESI) chamber in positive ion mode with quantification parameters as capillary $(3.50 \mathrm{kV})$, and collision energy $(58.0 \mathrm{eV})$. The operating conditions were set as; collision gas (Argon) with a pressure of $7.0 \times 10-3 \mathrm{~Pa}$ having desolvation temperature and desolvation gas flow of $400^{\circ} \mathrm{C}$ and $800 \mathrm{~L} / \mathrm{hr}$ respectively. The transition at $\mathrm{m} / \mathrm{z} 413.4 \rightarrow 194.2$ was adopted for quantification of ZP under the multiple reaction monitoring modes (MRM). The Mass-Lynx software (V4.1, SCN918) was used for the analysis of data obtained. ${ }^{18}$

\section{In vivo Pharmacodynamic Study of ZP-LPS \\ Experimental Design}

To evaluate the prolonged efficacy following a single administration of ZP-LPS depot formulation, experimental model of schizophrenia was developed by intraperitoneal injection of MK-801 (0.5 mg/kg, i.p.) as reported previously $^{19}$ and the Morris Water Maze (MWM) test was used for the evaluation of neurobehavioral assessment (memory and spatial learning). ${ }^{20,21}$

Twenty-four animals used in the study were randomly divided into 4 groups each containing 6 animals $(n=6)$. To 1$)$ healthy group: MK-801 was not injected and no treatment was given; 2) SCZ group: MK-801 was injected at day 0, day 7, day 28 and no treatment was given; 3) ZPS group: received subcutaneous ZP suspension and MK-801 was injected at day 0 and day 7; and 4) ZP-LPS group: received subcutaneous ZP-LPS formulation and MK-801 was injected at day 0 , day 7 , and day 28. Wherever required MK-801 was injected $1 \mathrm{~h}$ post administration of treatment. At 0,7 th and 28th day the probe trial was conducted and before $24 \mathrm{hrs}$ of each probe trial, acquisition trial was performed.

\section{Morris Water Maze (MWM) Test}

A circular black colored water pool, $0.60 \mathrm{~m}$ high and $1.50 \mathrm{~m}$ in diameter, was used as experimental set up. The circular tank was divided into four equal spaced quadrants viz. North-West (NW), South-West (SW), North-East (NE), and South-East (SE). The temperature of water in the pool was kept at $25 \pm 2{ }^{\circ} \mathrm{C}$. During the familiarization period (training session), a square shaped platform of $10 \mathrm{~cm}$ $\mathrm{x} 10 \mathrm{~cm}$ was placed approx one $\mathrm{cm}$ beneath the water surface and hidden at the center of target quadrant (NW). The rats were trained for 5 successive days ( 4 times in a day) before proceeding towards probe trial. The session was terminated when the rat found and climbed on to the platform or unable to locate the platform by $120 \mathrm{sec}$. After removing the platform from the maze, the probe trial was conducted $24 \mathrm{hrs}$ after the last training session for the spatial memory retention. The parameters such as the swim escape latency, time spent in the target quadrant and path length was evaluated using a video tracking system (software SMART v3.0.03, Harvard, U.S.).

\section{Statistical Analysis}

All the experimental data were statistically analyzed by one-way ANOVA and two-way ANOVA by Tukey's test and Benforinitest respectively. The $p$ value less than 0.05 was considered to be significant. All the results were showed as mean \pm standard deviation (SD).

\section{Results}

\section{Development and Optimization of ZP-LPS}

LPS formulation was prepared varying concentrations of PE80, MCT and ethanol. PE80 amount was varied from $40 \mathrm{mg}$ to $70 \mathrm{mg}$, MCT amount was varied from $15 \mathrm{mg}$ to $35 \mathrm{mg}$ and ethanol was fixed at $10 \mathrm{mg}$. All the developed formulations were clear transparent to translucent liquids of light yellow color with viscosity ranging from $50.4 \pm$ $2.7 \mathrm{cP}$ to $837.5 \pm 16.1 \mathrm{cP}$ as shown in Figure 1. High viscosities were observed for ZP-LPS containing high lipid ratios (approx 70\%). Further aqueous titration was done to observe the phase-transition of the formulation. Selection of formulation was based on ability of formulation to undergo phase-transition with minimum quantity of water.The results obtained were represented by pseudoternary phase diagram (Figure 2). Out of the various ratios tried, formulations with PE80: MCT in ratio of 50:35, $60: 15,60: 25,60: 35,70: 15 \quad 70: 25$ and $70: 35$ utilizes $10 \mathrm{mg}$ of aqueous phase to undergo phase transition. The results are in corroboration with previous studies where it was reported that phospholipid with concentrations less 


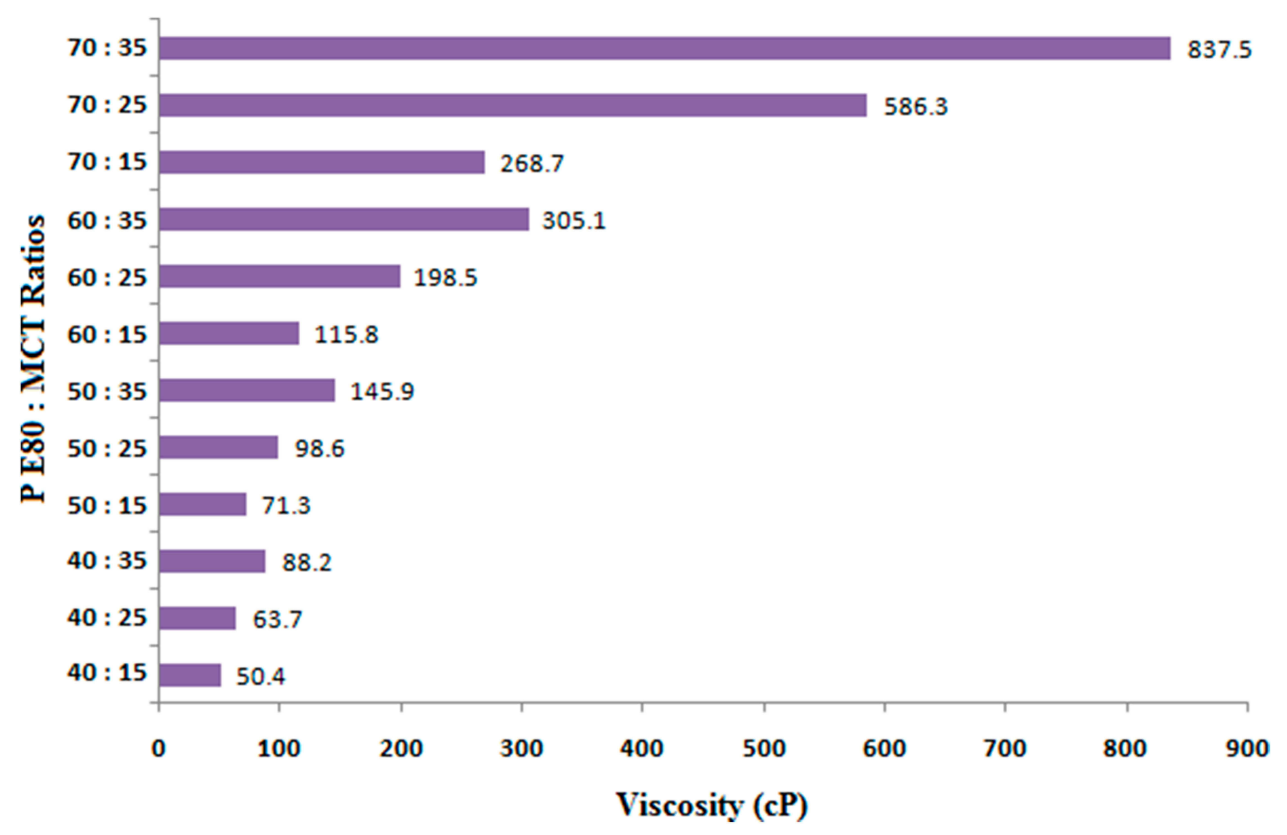

Figure I Viscosities of various ZP-LPSs formulated with varying ratios of P E80: MCT $(n=3)$.

than or equivalent to $50 \% \mathrm{w} / \mathrm{w}$ require high water content to undergo phase-transition. ${ }^{12,22}$ Additionally, formulations with PE80: MCT in ratio of 60:35, 70:25 and 70:35 were viscous (viscosity greater than $300 \mathrm{cP}$ ) and not suitable for subcutaneous injection. ${ }^{23}$ Thus, formulation with PE80: MCT in ratio of 50:35, 60:15, 60:25, and 70:15 were selected for rheological studies.

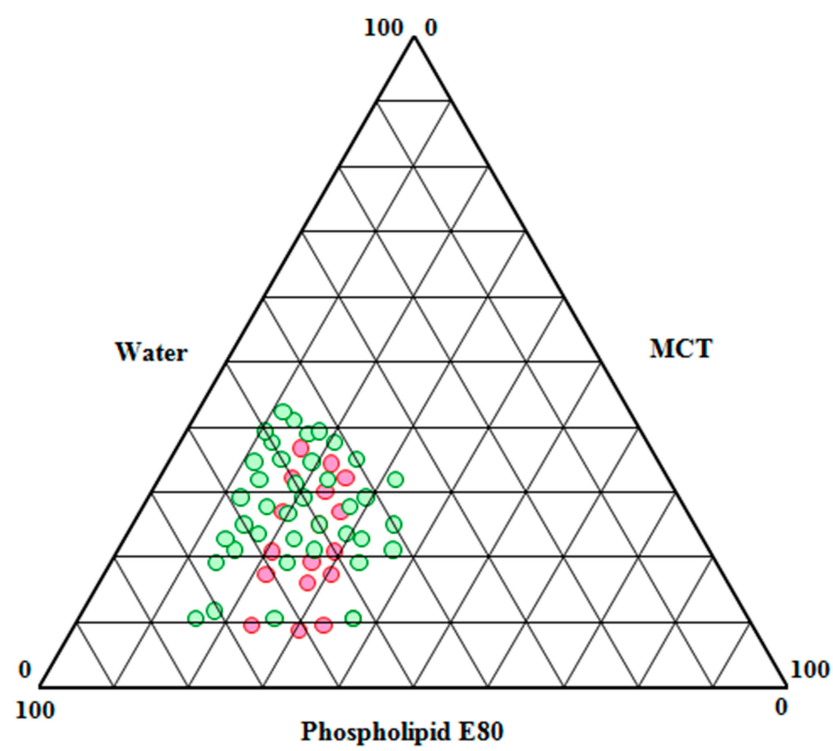

Figure 2 Pseudo-ternary phase diagram depicting quantity of water required by various formulated ZP-LPS for conversion from liquid to semi-solid gel $(n=3)$ (red dots indicate formulations having initial viscosity $>300 \mathrm{cP}$ ).

\section{Dynamic Rheological Measurements}

Dynamic viscosity determinations were done to determine onset time for gelation and gel strength of the selected ZPLPS formulations upon addition of $10 \mathrm{mg}$ PBS. Figure 3 shows change in viscosity with respect to time at constant frequency values. Out of the formulations tested, ZP-LPS with PE80: MCT ratio of 60:25 and 70:15 demonstrated rapid onset of gelation as shown by steep slope of the curve compared to other formulations. However, the viscosity change at any time point was maximum in ZP-LPS with PE80: MCT ratio of 60:25 indicating highest gel strength. Further, the percentage of cumulative drug release ( $\% \mathrm{CDR})$ at 1 day from ZP-LPS with PE80: MCT ratio of $60: 25$ and $70: 15$ were $10.83 \pm 1.3 \%$ and 1.7 $\pm 0.5 \%$ respectively. The low $\%$ CDR in case of $70: 15$ was could be due to highest phospholipid content which might act as a barrier in drug partitioning. ${ }^{24}$ Hence, after considering both the factors formulation with PE80: MCT ratio of 60:25 was considered optimized and was further evaluated.

\section{Solubility Study of ZP-LPS}

The results of the solubility studies showed that being crystalline and water insoluble, no ZP was dissolved in aqueous medium from ZP suspension. Interestingly, the solubility of $\mathrm{ZP}$ was found to be $28 \mu \mathrm{g} / \mathrm{mL}$ from ZP-LG formulation. This enhancement in the aqueous solubility of ZP is probably due to lipidic amorphization owing to complexation of 


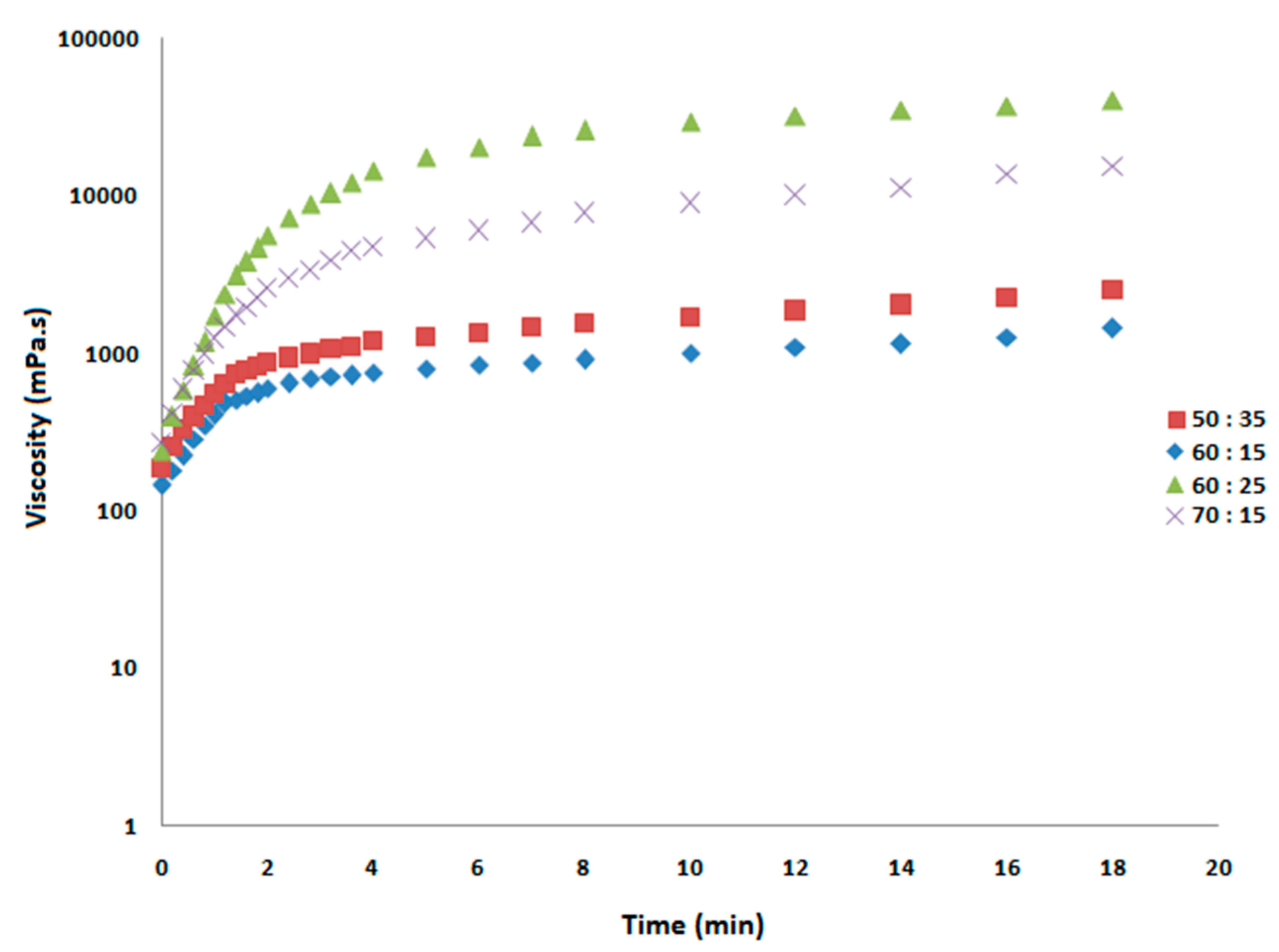

Figure 3 Dynamic rheological characterization of various prepared ZP-LPSs showing viscosity (mPa.s) vs time profile (min) at constant frequency ( $\mathrm{n}=3$ ).

ZP with phospholipid and MCT. Such an increase in solubility due to lipid amorphization has been previously reported for some other water insoluble drugs. ${ }^{25}$

\section{Drug Release Studies of ZP-LPS}

The drug release studies for ZP-LPS was performed using the dialysis method using PBS containing 1\% Tween 20 (pH 7.4) as the release medium and the comparison was done with $\mathrm{ZP}$ suspension. The results were shown in Figure 4. As expected, the percentage cumulative release of $\mathrm{ZP}$ from $\mathrm{ZP}$ suspension was $63.05 \pm 2.6 \%$ and $86.74 \pm 3.9 \%$, in $1 \mathrm{~h}$ and $2 \mathrm{~h}$, respectively. In contrast to this, ZP-LPS formulation demonstrated sustained release properties over period of 30 days with release of $10.8 \pm 1.3 \%, 42.5 \pm 4.7 \%, 73.4 \pm 5.6 \%$ and $88.5 \pm$ $5.3 \%$ at 1 day, 7 days, 14 days, and 30 days respectively. The phase transition of phospholipid to form gel structure, immiscibility of phospholipid in water and slow partitioning of lipophilic drug from ZP-LPS formulation to aqueous media might have played crucial role in sustaining drug release. ${ }^{13,26}$

\section{In vivo Biodegradation and}

\section{Biocompatibility Studies of ZP-LPS}

Visual observation and dimensional measurement was done to study biodegradation behavior of ZP-LPS formulation in rats. The images of depot formation at day 0 and day 28 are

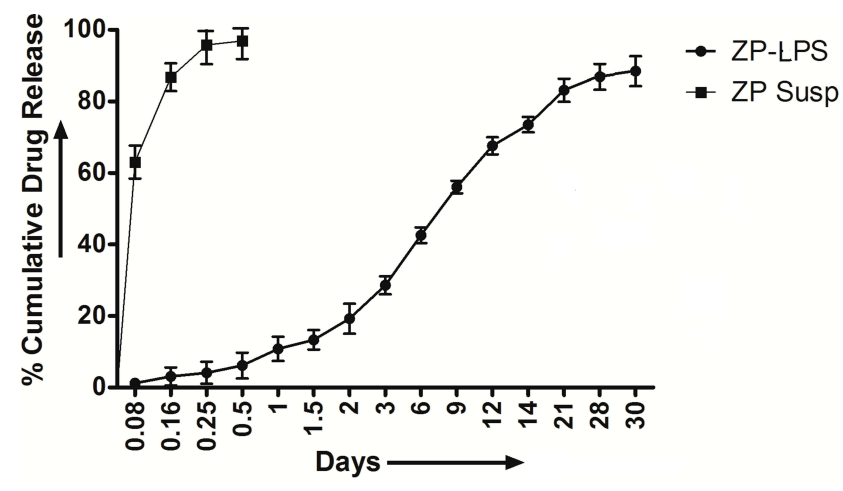

Figure 4 In vitro release profile of optimized ziprasidone loaded lipid phase-transition system (ZP-LPS) and ZP suspension in PBS release media containing I\% Tween $20(n=3)$.

shown in Figure 5. From the image at day 0 (Figure 5A) it was clearly evident that ZP-LPS solution rapidly forms gel (radius, $0.6 \mathrm{~cm}$ ) upon contact with subcutaneous aqueous fluid. At day 28 (Figure 5B), the gel depot size was markedly reduced (radius, $0.1 \mathrm{~cm}$ ) indicating gradually biodegradation with time. Further, no pathological changes and irritation was observed at site of injection suggesting that the developed ZP-LG formulation is safe and biocompatible.

\section{In vivo Depot Formation of ZP-LPS by Gamma Scintigraphy}

Gamma scintigraphy study was performed to visually confirm the formation of gel depot following subcutaneous 
Day 0

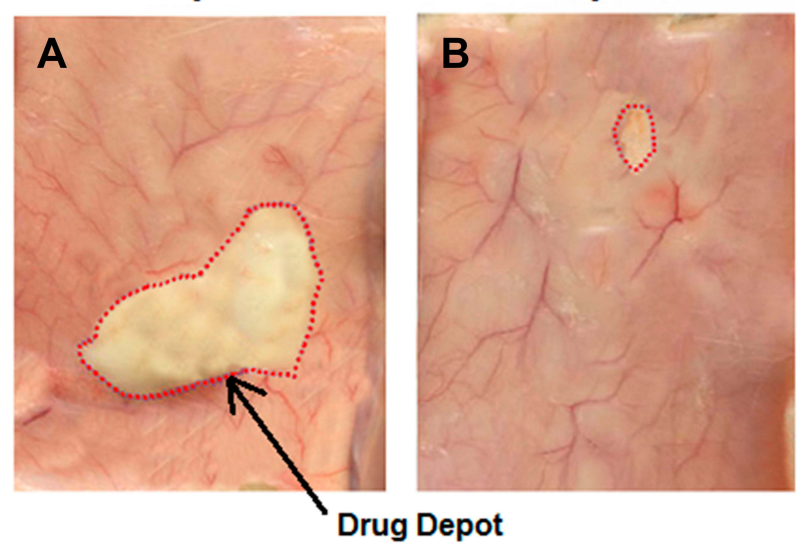

Figure 5 Biodegradation studies (A) showing changes at the site of injection and depot formation at day 0; (B) reduction in the size of depot after 28 days.

injection of liquid ZP-LPS formulation to rat. Following administration of radiolabel-ZP-LPS formulation, scintigraphic imaging was done at $15 \mathrm{~min}, 6 \mathrm{~h}$ and $12 \mathrm{~h}$ time point. Figure 6A showed that depot formation occurs within 15 mins post subcutaneous injection of liquid ZP-LPS and it remained intact throughout the study duration (Figure 6C). Spreading of radioactivity, as observed in Figure 6C, indicated release of radiolabel-ZP into the systemic circulation from the depot formulation. Due to the short half-life of technetium the study was not continued further.

\section{In vivo Pharmacokinetic Study of ZP-LPS}

Plasma levels of ZP in rats after subcutaneous injection of ZP suspension and ZP-LPS are shown in Figure 7. After subcutaneous administration of ZP suspension, ZP concentrations increased quickly and reached a maximum value of $1210.38 \mathrm{ng} / \mathrm{mL}$ within $1 \mathrm{~h}$, followed by a rapid clearance. In contrast, the maximum concentration of ZP after injection of ZP-LPS was $537.4 \pm 27.1 \mathrm{ng} / \mathrm{mL}$, which then gradually decreased but was maintained above the minimal therapeutic concentration for up to 28 days. The $t_{1 / 2}$ values of the ZPLPS and ZP suspensions were $486.73 \pm 17.61$ and $2.77 \pm 0.3$ $\mathrm{h}$, respectively. A paired $t$-test showed that pharmacokinetic parameters between ZP suspension and ZP-LPS had significant differences (Table 1). The nearly 45 times increased in AUC for ZP-LPS in comparison to ZP suspension and overall results of pharmacokinetic study demonstrate that ZPLPS formulation could be useful for prolong release of ZP.

\section{In vivo Pharmacodynamic Study of ZP-LPS}

The MWM test is a behavioral procedure used to measure the effect of neurocognitive disorders on spatial learning in rodents. The parameters measured are: 1) escape latency; 2) time spent in target quadrant; and 3) total distance traveled. The results of the MWM test conducted on day 0 , day 7, and day 28 are shown in Table 2.

\section{Effect of ZP-LPS on Escape Latency in MWM Test}

Escape latency is defined as time taken by rodent to reach the hidden platform. The SCZ group demonstrated a significantly prolonged escape latency $(\mathrm{p}<0.001)$ compared to the healthy group indicative of neurodegeneration following administration of MK 801. In the ZPS group, protective effects against

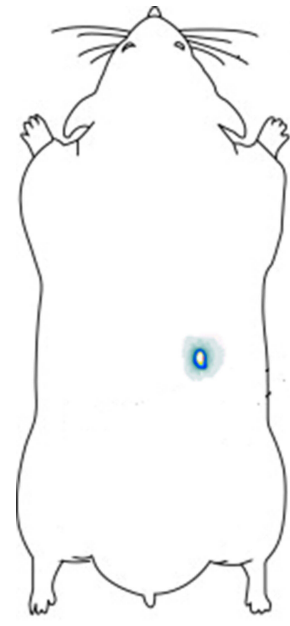

A

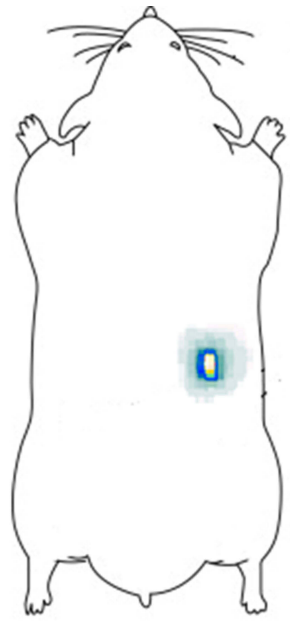

B

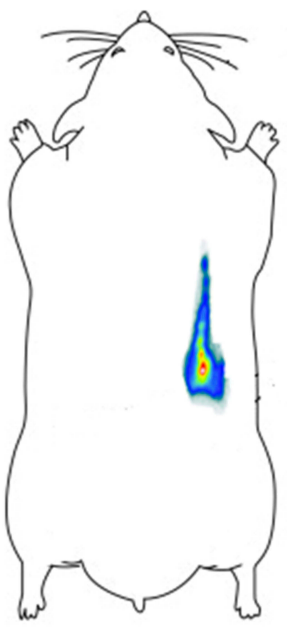

C

Figure 6 Gamma scintigraphic images after s.c. injection in rats (A) showing depot formation after 15 min; (B) after 6 h, depot remain intact; (C) after I2 h, spreading of radioactivity indicating release of radiolabel-ZP into systemic circulation. 


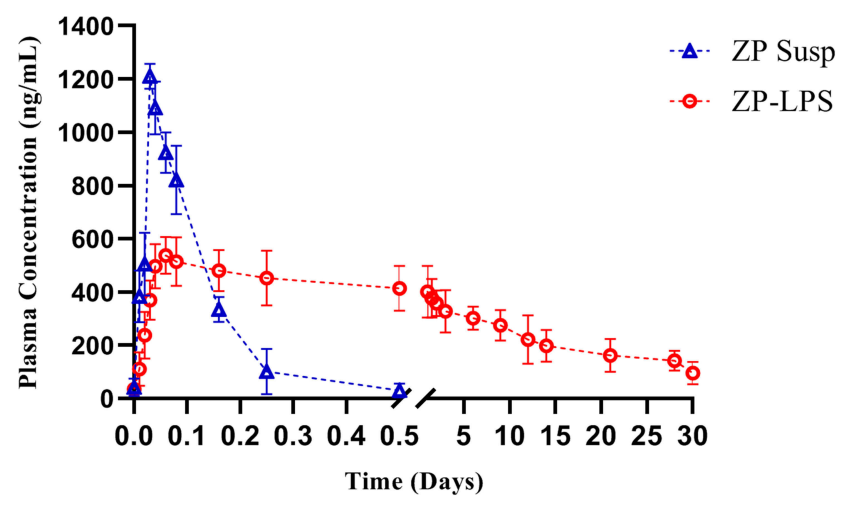

Figure 7 Plasma concentration vs time profile after a single subcutaneous injection of ZP suspension and ZP-LPS in rats $(n=6)$.

MK 801 were observed at day 0 as the escape latency time was significantly less than the SCZ group $(\mathrm{p}<0.001)$ and not significantly different from healthy group $(\mathrm{p}=0.2390)$. However, at day 7 , the escape latency time was not significantly different from the SCZ group $(\mathrm{p}=0.1809)$ indicating that $\mathrm{ZP}$ suspension was no more effective. This could be due to the inability of suspension to sustain drug release for prolonged periods and a short half-life $(\sim 2.5 \mathrm{~h})$ of ZP. Notably, the ZP-LPS group demonstrated escape latency times not significantly different ( $>0.05$ ) from healthy group at all the time points. While in comparison with the SCZ group, a significant reduction $(p<0.001)$ in escape latency time was found at day 0 , day 7 , and day 28 .

\section{Effect of ZP-LPS on Time Spent in Target Quadrant and Total Distance Traveled in MWM Test}

SCZ group demonstrated significant reduction in time spent $(\mathrm{p}<0.001)$ in target quadrant and total distance

Table I In Vivo Pharmacokinetic Parameters After Subcutaneous Injection of ZP Suspension and Developed ZPLPS System $(n=6)$

\begin{tabular}{|l|l|l|}
\hline \multirow{2}{*}{ Pharmacokinetic Parameters } & \multicolumn{2}{|c|}{ Groups } \\
\cline { 2 - 3 } & ZP Suspension & ZP-LPS \\
\hline $\mathrm{C}_{\max }(\mathrm{ng} / \mathrm{mL})$ & $1210.38 \pm 46.9$ & $537.4 \pm 27.1^{\mathrm{a}}$ \\
$\mathrm{T}_{\max }(\mathrm{h})$ & $0.72 \pm 0.2$ & $1.44 \pm 0.4^{\mathrm{b}}$ \\
$\mathrm{K}_{\mathrm{e}}\left(\mathrm{h}^{-1}\right)$ & $0.2260 \pm 0.09$ & $0.00142 \pm 0.01^{\mathrm{a}}$ \\
$\mathrm{t}_{\mathrm{l} / 2}(\mathrm{~h})$ & $3.06 \pm 0.73$ & $486.73 \pm 17.61^{\mathrm{a}}$ \\
AUC $_{0-\mathrm{t}}(\mathrm{ng} . \mathrm{h} / \mathrm{mL})$ & $3624.14 \pm 137.6$ & $166,695.07 \pm 1805.9^{\mathrm{a}}$ \\
AUC $_{0-\infty}(\mathrm{ng} . \mathrm{h} / \mathrm{mL})$ & $3756.85 \pm 125.1$ & $233,419.09 \pm 2142.7^{\mathrm{a}}$ \\
\hline
\end{tabular}

Notes: $\mathrm{AUC}_{0-\mathrm{t}}$, area under the concentration-time curve from time 0 to time ' $\mathrm{t}$ '; $\mathrm{AUC}_{0-\infty}$, area under the concentration-time curve from time 0 to infinity; $\mathrm{C}_{\max }$, maximum observed serum concentration; Ke, elimination rate constant; $T_{\max }$, time to maximum serum concentration; $t_{1 / 2}$, terminal phase half-life. All data presented as mean $\pm S D$. ${ }^{a} p<0.000$ I when compared with $Z P$ suspension; ${ }^{b} p<0.001$ when compared with ZP suspension. traveled $(\mathrm{p}<0.001)$ compared to healthy group confirming neurodegeneration following MK 801 administration. ZPS group showed significant increase $(\mathrm{p}<0.001)$ in both time spent and total distance traveled in target quadrant from SCZ group at day 0 . However, the results were not significantly different $(\mathrm{p}>0.05)$ on day 7 from $\mathrm{SCZ}$ group. As expected, ZP-LPS group demonstrated time spent and total distance traveled in target quadrant results similar to healthy group ( $p>0.05)$ and significantly different from SCZ group ( $p-<-0.001)$ at all the tested days. This probably was due to sustained drug release from the ZP-LPS formulation over period of 28 days as demonstrated by drug release, in vivo biodegradation and pharmacokinetic studies.

\section{Discussion}

An LPS based on phospholipid, MCT and ethanol was developed for the sustained parenteral delivery of $\mathrm{ZP}$ for the management of schizophrenia. After contact with aqueous fluids, the phospholipid and MCT in the LPS realign and a mesophase forms spontaneously, creating a drug release depot at the injection site. ${ }^{14}$ Presence of low percentage of ethanol as water-miscible organic solvent, keeps the LPS in a low-viscosity injectable state before the phase transition. ${ }^{22}$ The method used for the preparation of LPS was simple and required mixing components for 30 min giving rise to a liquid formulation for injection. Based on the fact that subcutaneous tissue contains a low amount of water and sustained drug release depends on gel strength, the formulation was optimized based on: 1) initial viscosity; 2) phase transition in contact with minimum quantity of water; 3) rapid gelation; 4) gel strength; and 5) \% CDR at 1 day.

At first, ZP-LPS formulations were prepared varying ratios of PE80 and MCT. Formulations were then titrated with PBS as an aqueous medium to depict changes in viscosity via pseudo-ternary phase diagram (Figure 2). ZP-LPS formulations, having an initial viscosity less than $300 \mathrm{cP}$, phase-transit to gel with the lowest amount of PBS were selected for further investigation. The final selection of the ZP-LPS formulation was done based on the onset time of gelation, gel strength and drug release behavior. Dynamic rheology showed that formulation with PE80: an MCT ratio of 60:25 and 70:15 showed rapid onset and highest gel strength (Figure 3). However, formulation formed with 70:15, PE80: MCT was unable to release the drug (approx $2 \%$ ) at day 1 , possibly due to the high amount of phospholipid that acted as a barrier for the partitioning of lipophilic 
Table 2 In Vivo Pharmacodynamic Studies Results and Effect of Developed ZP-LPS on Different MWM Test Parameters ( $\mathrm{n}=6$ )

\begin{tabular}{|c|c|c|c|c|c|c|c|c|c|}
\hline \multirow[t]{3}{*}{ Groups } & \multicolumn{9}{|c|}{ MWM Test Parameters } \\
\hline & \multicolumn{3}{|c|}{ Escape Latency (s) } & \multicolumn{3}{|c|}{ Time Spent in Target Quadrant (s) } & \multicolumn{3}{|c|}{ Total Distance Traveled (cm) } \\
\hline & Day 0 & Day 7 & Day 28 & Day 0 & Day 7 & Day 28 & Day 0 & Day 7 & Day 28 \\
\hline Healthy & $36.18 \pm 1.97$ & $34.90 \pm 2.13$ & $35.84 \pm 1.50$ & $34.27 \pm 2.07$ & $34.52 \pm 1.61$ & $34.62 \pm 1.34$ & $315.6 \pm 6.06$ & $310.7 \pm 8.57$ & $308.6 \pm 6.10$ \\
\hline SCZ & $68.19 \pm 3.13^{\mathrm{a}}$ & $67.37 \pm 2.08^{\mathrm{a}}$ & $67.88 \pm 1.31^{\mathrm{a}}$ & $20.45 \pm 1.14^{\mathrm{a}}$ & $20.30 \pm 1.20^{\mathrm{a}}$ & $20.21 \pm 1.98^{\mathrm{a}}$ & $514.4 \pm 7.96^{\mathrm{a}}$ & $521.1 \pm 6.28^{\mathrm{a}}$ & $526.2 \pm 5.73^{\mathrm{a}}$ \\
\hline ZPS & $40.45 \pm 4.97^{\mathrm{b}, \mathrm{c}}$ & $64.99 \pm 1.46^{\mathrm{a}, \mathrm{d}}$ & n.d & $32.83 \pm 1.49^{\mathrm{b}, \mathrm{c}}$ & $21.18 \pm 1.24^{\mathrm{a}, \mathrm{d}}$ & n.d & $383.2 \pm 5.68^{\mathrm{b}, \mathrm{c}}$ & $5|I .13 \pm 7.4|^{\mathrm{a,d}}$ & n.d \\
\hline ZP-LPS & $41.95 \pm 1.85^{\mathrm{b}, \mathrm{c}}$ & $40.80 \pm 2.54^{\mathrm{b}, \mathrm{c}}$ & $40.32 \pm 1.86^{\mathrm{b}, \mathrm{c}}$ & $31.05 \pm 1.36^{\mathrm{b}, \mathrm{c}}$ & $32.12 \pm 3.49^{\mathrm{b}, \mathrm{c}}$ & $32.43 \pm 1.76^{\mathrm{b}, \mathrm{c}}$ & $362.6 \pm 7.35^{\mathrm{b}, \mathrm{c}}$ & $376.4 \pm 6.42^{\mathrm{b}, \mathrm{c}}$ & $381.03 \pm 6.83^{\mathrm{b}, \mathrm{c}}$ \\
\hline
\end{tabular}

Notes: n.d, not detected; ${ }^{a} p<0.00$ I when compared with healthy group; ${ }^{b} p>0.05$ when compared with healthy group; ${ }^{c} p<0.00$ I when compared with SCZ group; ${ }^{d} p>0.05$ when compared with SCZ group. All data expressed as mean \pm SD.

ZP. Such observations have been previously reported. ${ }^{24}$ Formulations require an additional intramuscular injection of ZP making the therapy inconvenient. ZP-LPS with PE80: MCT ratio of 60:25 showed sustained drug release over period of 30 days without burst effect. The sustained drug release was due to lipid phase transition and slows partitioning of lipophilic drug to aqueous media. The absence of burst effect is due to rapid gelation, higher gel strength and viscous changes as demonstrated by dynamic rheological measurements. The phase transition results in depot formation. The depot formation was proved by in vivo scintigraphy studies which showed stable depot for $12 \mathrm{~h}$ (Figure 6). In vivo studies revealed that depot formation occurs within 15 min of subcutaneous application of ZP-LPS formulation. Further, visual and dimensional analysis showed that the depot is biodegradable (Figure 5). Absence of irritation and pathological change indicates that the ZP-LPS formulation is biocompatible and safe for parenteral administration.

In pharmacokinetic study, ZP-LPS showed a significantly lower $\mathrm{C}_{\max }$ value than that found for the $\mathrm{ZP}$ suspension, indicating that the initial burst of ZP-LPS was much lower than that of ZP suspension (Figure 7 and Table 1). The percentage of ethanol in the pre-gel solution was limited, thereby causing a less significant initial burst. ${ }^{13}$ On the other hand, the $t_{1 / 2}$ value of the ZP-LPS was much longer than that of the $Z P$ suspension, indicating a sustained release of ZP from ZP-LPS. The overall results of the pharmacokinetic study demonstrate that ZP-LPS formulation could be useful for prolonged delivery of $Z \mathrm{P}$ over a 1 month period via single subcutaneous injection.

Following this, pharmacodynamic studies were conducted using MWM Test to evaluate the efficacy of developed ZP-LPS formulation. An experimental model of schizophrenia was developed by intraperitoneal injection of MK-801 (0.5 mg/kg, i.p.). ${ }^{19}$ The result of the current study showed that the administration of MK-801 induced significant cognitive impairment which was observed by the estimation of escape latency time, time spent in target quadrant, and total distance traveled. These parameters were found to be significantly derailed upon administration of MK-801 when compared with healthy group (Table 2). Treatment with ZP suspension was found to be ineffective in reversing these attributes of cognitive dysfunction, except at day 0 , which could be explained by the fact that $\mathrm{ZP}$ suspension was unable to sustain the release of $\mathrm{ZP}$ and only effective at day 0 . On the other hand, the ZP-LPS formulation effectively reversed the aforementioned parameters towards normal when compared with SCZ group (MK-801) over period of 28 days. This indicates protective efficacy of single administration of ZP-LPS system against schizophrenia.

\section{Conclusion}

A novel parenteral lipid based phase-transition system was developed using phospholipid and MCT in ethanol for the sustained release of ZP. ZP-LPS showed sustained in vitro and in vivo release of $\mathrm{ZP}$ with a small initial burst. The gamma scintigraphic evaluation after s.c. injection of ZPLPS depicts depot formation within 15 min. Whereas, biodegradation studies proved depot degradation over a period of 28 days and revealed its safety and biocompatibility with surrounding tissues. The pharmacokinetic study showed sustained release of ZP from ZP-LPS over a period of 1 month. In efficacy evaluation using the MWM test, ZP-LPS technology produced protective effect against MK-801 induced schizophrenia for 28 days. In the ZP-LPS group all three parameters escape latency, time spent in target quadrant and total distance traveled were found to be significantly different $(\mathrm{p}<0.001)$ from the MK801 (SCZ) group when tested at day 0, day 7, and day 28 . Hence, it may be used as a promising depot forming LPS to improve patient compliance in schizophrenia therapy. 


\section{Highlights}

- ZP-LPS were developed.

- ZP-LPS possess sustained release properties both in vitro and in vivo.

- ZP-LPS are safe and biodegradable.

- A single dose ZP-LPS showed a protective effect against schizophrenia for a period of 28 days.

\section{Abbreviations}

EPs, extrapyramidal side effects; h, hour; LPS, lipid phase-transition system; MCT, medium chain triglyceride; MWM, Morris Water Maze test; HPLC, high-performance liquid chromatography; i.p, intra peritoneal; PBS, phosphate-buffered saline solution; P E80, phospholipid E 80; s.c, subcutaneous; SD, standard deviation; ZP, ziprasidone; ZP-LPS, ziprasidone loaded lipid phase-transition system.

\section{Acknowledgment}

The authors of this research work sincerely grateful to the UGC (University Grants Commission), New Delhi, India for financial support (File no. F1-17.1/2015-16/MANF2015-17-DEL-64730) throughout this study.

\section{Disclosure}

The authors report no conflicts of interest in this work.

\section{References}

1. Nahata T, Saini TR. Optimization of formulation variables for the development of long acting microsphere based depot injection of olanzapine. J Microencapsul. 2008;25(6):426-433. doi:10.1080/ 02652040802033913

2. Lindenmayer JP, Khan A. Pharmacological treatment strategies for schizophrenia. Expert Rev Neurother. 2004;4(4):705-723. doi:10.1586/14737175.4.4.705

3. Schmidt AW, Lebel LA, Howard HR, Zorn SH. Ziprasidone: a novel antipsychotic agent with a unique human receptor binding profile. Eur J Pharmacol. 2001;425(3):197-201. doi:10.1016/S0014-2999(01) 01188-8

4. Ebdrup BH, Rasmussen H, Arnt J, Glenthøj B. Serotonin 2A receptor antagonists for treatment of schizophrenia. Exp Opin Investig Drugs. 2011;20(9):1211-1223. doi:10.1517/13543784.2011.601738

5. Richtand NM, Welge JA, Logue AD, Keck PE, Strakowski SM, McNamara RK. Dopamine and serotonin receptor binding and antipsychotic efficacy. Neuropsychopharmacology. 2007;32 (8):1715-1726. doi:10.1038/sj.npp.1301305

6. Lesem MD, Zajecka JM, Swift RH, Reeves KR, Harrigan EP. Intramuscular ziprasidone, $2 \mathrm{mg}$ versus $10 \mathrm{mg}$, in the short-term management of agitated psychotic patients. $J$ Clin Psychiatry. 2001;62(1):12-18. doi:10.4088/JCP.v62n0104

7. Miceli JJ, Wilner KD, Swan SK, Tensfeldt TG. Pharmacokinetics, safety, and tolerability of intramuscular ziprasidone in healthy volunteers. J Clin Pharmacol. 2005;45(6):620-630. doi:10.1177/ 0091270005276485
8. Caley CF, Cooper CK. Ziprasidone: the fifth atypical antipsychotic. Ann Pharmacother. 2002;36(5):839-851. doi:10.1345/aph.1A053

9. Rosa AR, Franco C, Torrent C, et al. Ziprasidone in the treatment of affective disorders: a review. CNS Neurosci Ther. 2008;14:278-286. doi:10.1111/j.1755-5949.2008.00056.x

10. Cunningham Owens DG, Carroll A, Fattah S, Clyde Z, Coffey I, Johnstone EC. A randomized, controlled trial of a brief interventional package for schizophrenic out-patients. Acta Psychiatr Scand. 2001;103(5):362-369. doi:10.1034/j.1600-0447.2001.00 132.x

11. Pennington $\mathrm{M}$, McCrone $\mathrm{P}$. The cost of relapse in schizophrenia. Pharmacoeconomics. 2017;35(9):921-936. doi:10.1007/s40273-0170515-3

12. Li H, Liu T, Zhu Y, et al. An in situ-forming phospholipid-based phase transition gel prolongs the duration of local anesthesia for ropivacaine with minimal toxicity. Acta Biomater. 2017;58:136-145. doi:10.1016/j.actbio.2017.06.013

13. Zhang T, Peng Q, San FY, et al. A high-efficiency, low-toxicity, phospholipids-based phase separation gel for long-term delivery of peptides. Biomaterials. 2015;45:1-9. doi:10.1016/j.biomaterials.2014. 12.042

14. Han L, Xue J, Wang L, et al. An injectable, low-toxicity phospholipid-based phase separation gel that induces strong and persistent immune responses in mice. Biomaterials. 2016;105:185-194. doi:10.1016/j.biomaterials.2016.08.007

15. Pavlovic M, Malesevic M, Nikolic K, Agbaba D. Development and validation of an HPLC method for determination of ziprasidone and its impurities in pharmaceutical dosage forms. J AOAC Int. 2011;94 (3):713-722. doi:10.1093/jaoac/94.3.713

16. Banerjee T, Singh AK, Sharma RK, Maitra AN. Labeling efficiency and biodistribution of Technetium- $99 \mathrm{~m}$ labeled nanoparticles: interference by colloidal tin oxide particles. Int J Pharm. 2005;289(1-2):189-195. doi:10.1016/j.ijpharm.2004.09.022

17. Malik A, Khar R, Ali A, Bhatnagar A, Mittal G, Gupta H. Physiologically active hydrogel (in situ gel) of sparfloxacin and its evaluation for ocular retention using gamma scintigraphy. $J$ Pharm Bioallied Sci. 2015;7(3):195. doi:10.4103/0975-7406.160015

18. Sreedasyam R, Akulathukaram B, Khagga M, et al. Sensitive LC-MS/MS method for the determination of ziprasidone in human plasma and its pharmacokinetic application. Int J Chem Anal Sci. 2012;3(313):1439-1445.

19. Tanyeri P, Buyukokuroglu ME, Mutlu O, et al. Effects of ziprasidone, SCH23390 and SB277011 on spatial memory in the Morris water maze test in naive and MK-801 treated mice. PharmacolBiochemBehav. 2015. doi:10.1016/j.pbb.2015.09.014

20. Bromley-Brits K, Deng Y, Song W. Morris water maze test for learning and memory deficits in alzheimer's disease model mice. $J$ Vis Exp. 2011;(53). doi:10.3791/2920

21. Husain I, Akhtar M, Madaan T, et al. Tannins enriched fraction of emblica officinalis fruits alleviates high-salt and cholesterol diet-induced cognitive impairment in rats via Nrf2-ARE pathway. Front Pharmacol. 2018;9 (JAN):1-15. doi:10.3389/fphar.2018.00023

22. Wang D, Zhao J, Liu X, et al. Parenteral thermo-sensitive organogel for schizophrenia therapy, in vitro and in vivo evaluation. Eur J Pharm Sci. 2014;60:40-48. doi:10.1016/j.ejps.2014.04. 020

23. Xuan JJ, Yan YD, Oh DH, Choi YK, Yong CS, Choi HG. Development of thermo-sensitive injectable hydrogel with sustained release of doxorubicin: rheological characterization and in vivo evaluation in rats. Drug Deliv. 2011;18(5):305-311. doi:10.3109/ 10717544.2010.544690

24. Clay AT, Sharom FJ. Lipid bilayer properties control membrane partitioning, binding, and transport of P-glycoprotein substrates. Biochemistry. 2013;52(2):343-354. doi:10.1021/bi301532c 
25. Patel KK, Gade S, Anjum MM, et al. Effect of penetration enhancers and amorphization on transdermal permeation flux of raloxifene-encapsulated solid lipid nanoparticles: an ex vivo study on human skin. Appl Nanosci. 2019;9(6):1383-1394. doi:10.1007/ s13204-019-01004-6
26. Weng Larsen S, Larsen C. Critical factors influencing the in vivo performance of long-acting lipophilic solutions-impact on in vitro release method design. AAPS J. 2009;11(4):762-770. doi:10.1208/ s12248-009-9153-9

\section{Publish your work in this journal}

Drug Design, Development and Therapy is an international, peerreviewed open-access journal that spans the spectrum of drug design and development through to clinical applications. Clinical outcomes, patient safety, and programs for the development and effective, safe, and sustained use of medicines are a feature of the journal, which has also been accepted for indexing on PubMed Central. The manuscript management system is completely online and includes a very quick and fair peer-review system, which is all easy to use. Visit http://www. dovepress.com/testimonials.php to read real quotes from published authors.

Submit your manuscript here: https://www.dovepress.com/drug-design-development-and-therapy-journal 